\title{
An On-Treatment Analysis of the MARQUIS Study: Interventions to Improve Inpatient Medication Reconciliation
}

\author{
Amanda S Mixon, MD, MS, MSPH, FHM1,2,3*, Sunil Kripalani, MD, MSc, SFHM ${ }^{2,3}$, Jason Stein, MD, SFHM ${ }^{4}$, \\ Tosha B Wetterneck, MD, MS, FACP, SFHM ${ }^{5}$, Peter Kaboli, MD, MS, FACP, FHM ${ }^{6}$, Stephanie Mueller, MD, MPH7,8, \\ Elisabeth Burdick, MS', Nyryan V Nolido, MA8, Stephanie Labonville, PharmD, BCPS ${ }^{10}$, \\ Jacquelyn A Minahan ${ }^{8,11}$, E John Orav, PhD ${ }^{8,12}$, Jenna Goldstein, MA ${ }^{13}$, Jeffrey L Schnipper, MD, MPH78,9
}

\begin{abstract}
${ }^{1}$ GRECC, VA Tennessee Valley Healthcare System, Vanderbilt University Medical Center, Nashville, Tennessee; ${ }^{2}$ Section of Hospital Medicine, Vanderbilt University Medical Center, Nashville, Tennessee; ${ }^{3}$ Center for Clinical Quality and Implementation Research, Vanderbilt University Medical Center, Nashville, Tennessee; ${ }^{4}$ Section of Hospital Medicine, Emory University School of Medicine, Atlanta, Georgia, and 1Unit, Atlanta, Georgia; ${ }^{5}$ Department of Medicine, University of Wisconsin School of Medicine and Public Health, Madison, Wisconsin; ${ }^{6}$ Center for Access Delivery Research and Evaluation, lowa City VA Healthcare System, lowa City, lowa, and Division of General Internal Medicine, University of lowa Carver College of Medicine, lowa City, lowa; ${ }^{7}$ Hospital Medicine Unit, Brigham Health, Boston, Massachusetts; ${ }^{8}$ Division of General Internal Medicine and Primary Care, Brigham and Women's Hospital, Boston, Massachusetts; ${ }^{9}$ Harvard Medical School, Boston, Massachusetts; ${ }^{10}$ Department of Pharmacy, Brigham and Women's Hospital, Boston, Massachusetts; ${ }^{11}$ University of Kansas, Lawrence, Kansas; ${ }^{12}$ Harvard T.H. Chan School of Public Health, Boston, Massachusetts; ${ }^{13}$ Society of Hospital Medicine, Philadelphia, Pennsylvania.
\end{abstract}

It is unclear which medication reconciliation interventions are most effective at reducing inpatient medication discrepancies. Five United States hospitals' interdisciplinary quality improvement (Ql) teams were virtually mentored by $\mathrm{Ql}$-trained physicians. Sites implemented one to seven evidence-based interventions in 791 patients during the 25-month implementation period. Three interventions were associated with significant decreases in potentially harmful discrepancy rates: (1) defining clinical roles and responsibilities, (2) training, and (3) hiring staff to perform discharge medication reconciliation. Two interventions were associated with significant increases in potentially harmful discrepancy rates: training staff to take medication histories and implementing a new electronic health record (EHR). Hospitals should focus first on hiring and training pharmacy staff to assist with medication reconciliation at discharge and delineating roles and responsibilities of clinical staff. We caution hospitals implementing a large vendor EHR, as medication discrepancies may increase. Finally, the effect of medication history training on discrepancies needs further study. Journal of Hospital Medicine 2019;14:614-617. () 2019 Society of Hospital Medicine $\bigcup$ nintentional medication discrepancies in the hospital setting are common and contribute to adverse drug events, resulting in patient harm. ${ }^{1}$ Discrepancies can be resolved by implementing high-quality medication reconciliation, but there are insufficient data to guide hospitals as to which interventions are most effective at improving medication reconciliation processes and reducing harm. ${ }^{2}$ We recently reported that implementation of a best practices toolkit reduced total medication discrepancies in the Multi-Center Medication Reconciliation Quality Improvement Study (MARQUIS). ${ }^{3}$ This report describes the effect of individual toolkit components on rates of medication discrepancies with the potential for patient harm.

\section{METHODS}

Detailed descriptions of the intervention toolkit and study design of MARQUIS are published. ${ }^{4,5}$ Briefly, MARQUIS was a

\footnotetext{
${ }^{*}$ Corresponding Author: Amanda S. Mixon, MD, MS, MSPH, FHM; E-mail: Amanda.S.Mixon@vumc.org; Telephone: 615-936-3710; Twitter: @mixovida. Published online first August 21, 2019

Received: February 12, 2019; Revised: May 24, 2019; Accepted: May 30, 2019

() 2019 Society of Hospital Medicine DOI 10.12788/jhm.3308
}

pragmatic, mentored, quality improvement (Ql) study in which five hospitals in the United States implemented interventions from a best practices toolkit to improve medication reconciliation on noncritical care medical and surgical units from September 2011 to July 2014. We used a mentored implementation approach, in which each site identified the leaders of their local quality improvement team (ie, mentees) who received mentorship from a trained physician with $\mathrm{Ql}$ and medication safety experience. ${ }^{6}$ Mentors conducted monthly calls with their mentees and two site visits. Sites adapted and implemented one or more components from the MARQUIS toolkit, a compilation of evidence-based best practices in medication reconciliation. ${ }^{5,7}$

The primary outcome was unintentional medication discrepancies in admission and discharge orders with the potential for causing harm, as previously described. ${ }^{4}$ Trained study pharmacists at each site took "gold standard" medication histories on a random sample of up to 22 patients per month. These medications were then compared with admission and discharge medication orders, and all unintentional discrepancies were identified. The discrepancies were then adjudicated by physicians blinded to the treatment arm, who confirmed whether discrepancies were unintentional and carried the potential for patient harm. 
TABLE 1. Implementation of Components by Site

\begin{tabular}{|c|c|c|c|c|c|}
\hline Intervention Component & \multicolumn{5}{|c|}{ Site } \\
\hline \multicolumn{6}{|l|}{ Best Possible Medication History-Taking } \\
\hline Trained existing staff to take best possible medication histories & & $x$ & $x$ & $x$ & $x$ \\
\hline Hired additional staff to take best possible medication histories & & $x$ & $x$ & $x$ & \\
\hline Hired additional staff to perform discharge medication reconciliation and patient counseling & & & $x$ & & $x$ \\
\hline \multicolumn{6}{|l|}{ Roles and Responsibilities } \\
\hline Clearly defined roles and responsibilities and communicated this with clinical staff & & & & $x$ & \\
\hline \multicolumn{6}{|l|}{ Risk Stratification } \\
\hline Made improvements to existing medication reconciliation health information technology & & $x$ & & & $x$ \\
\hline \multicolumn{6}{|l|}{ Access to Medication Sources } \\
\hline Improved access to preadmission medication sources & & $x$ & & $x$ & \\
\hline
\end{tabular}

We employed a modification of a stepped wedge methodology to measure the incremental effect of implementing nine different intervention components, introduced at different sites over the course of the study, on the number of potentially harmful discrepancies per patient. These analyses were restricted to the postimplementation period on hospital units that implemented at least one intervention. All interventions conducted at each site were categorized by component, including dates of implementation. Each intervention component could be applied more than once per site (eg, when involving a new group of providers) or implemented on a new hospital unit or service, in which case, all dates were included in the analysis. We conducted a multivariable Poisson regression (with time divided into months) adjusted for patient factors, season, and site, with the number of potentially harmful discrepancies as the dependent variable, and the total number of gold standard medications as a model offset. The model was designed to analyze changes in the $y$-intercept each time an intervention component was either implemented or spread and assumed the change in the $y$-intercept was the same for each of these events for any given component. The model also assumes that combinations of interventions had independent additive effects.

\section{RESULTS}

Across the five participating sites, 1,648 patients were enrolled from September 2011 to July 2014. This number included 613 patients during the preimplementation period and 1,035 patients during the postimplementation period, of which 791 were on intervention units and comprised the study population. Table 1 displays the intervention components implemented by site. Sites implemented between one and seven components. The most frequently implemented intervention component was training existing staff to take the best possible medication histories (BPMHs), implemented at four sites. The regression results are displayed in Table 2 . Three interventions were associated with significant decreases in potentially harmful discrepancy rates: (1) clearly defining roles and responsibilities and communicating this with clinical staff (hazard ratio [HR] $0.53,95 \% \mathrm{Cl}$ : 0.32-0.87); (2) training existing staff to perform discharge medication reconciliation and patient counseling ( $\mathrm{HR} 0.64$, 95\% Cl: 0.46-0.89); and (3) hiring additional staff to perform discharge medication reconciliation and patient counseling (HR 0.48, 95\% Cl: 0.31-0.77). Two interventions were associated with significant increases in potentially harmful discrepancy rates: training existing staff to take BPMHs (HR 1.38, 95\% Cl: 1.21-1.57) and implementing a new electronic health record (EHR; HR 2.21, 95\% Cl: 1.64-2.97).

\section{DISCUSSION}

We noted that three intervention components were associated with decreased rates of unintentional medication discrepancies with potential for harm, whereas two were associated with increased rates. The components with a beneficial effect were not surprising. A prior qualitative study demonstrated the confusion related to clinicians' roles and responsibilities during medication reconciliation; therefore, clear delineations should reduce rework and improve the medication reconciliation process. ${ }^{8}$ Other studies have shown the benefits of pharmacist involvement in the inpatient setting, particularly in reducing errors at discharge. ${ }^{9}$ However, we did not anticipate that training staff to take BPMHs would be detrimental. Possible reasons for this finding that are based on direct observations by mentors at site visits or noted during monthly calls include (1) training personnel on this task without certification of competency may 
TABLE 2. Relationship between Potentially Harmful Medication Discrepancies Per Patient and Intervention Components by Site

\begin{tabular}{|c|c|c|}
\hline Intervention Component & Adjusted Incidence Rate Ratio ${ }^{a}\left(95 \% \mathrm{Cl}^{\mathrm{b}}\right)$ & $P$ Value \\
\hline Trained existing staff to take best possible medication histories & $1.38(1.21$ to 1.57$)$ & $<.001$ \\
\hline Hired additional staff to take best possible medication histories & $0.98(0.58$ to 1.65$)$ & .94 \\
\hline Trained existing staff to perform discharge medication reconciliation and patient counseling & $0.64(0.46$ to 0.89$)$ & .007 \\
\hline Hired additional staff to perform discharge medication reconciliation and patient counseling & $0.48(0.31$ to 0.77$)$ & .002 \\
\hline Clearly defined roles and responsibilities and communicating this with clinical staff & $0.53(0.32$ to 0.87$)$ & .01 \\
\hline Performed high-intensity interventions on high-risk patients & $1.28(0.89$ to 1.85$)$ & .18 \\
\hline Implemented a new electronic medical record & $2.21(1.64$ to 2.97$)$ & $<.001$ \\
\hline Made improvements to existing medication reconciliation health information technology & $0.82(0.51$ to 1.30$)$ & .40 \\
\hline Improved access to pre-admission medication sources & $1.42(0.46$ to 4.38$)$ & .54 \\
\hline \multicolumn{3}{|c|}{ 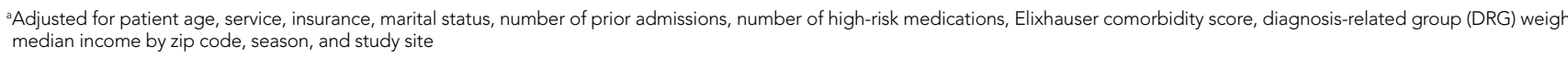 } \\
\hline \multicolumn{3}{|c|}{ b95\% confidence interval } \\
\hline
\end{tabular}

not sufficiently improve their skills, leading instead to diffusion of responsibility; (2) training personnel without sufficient time to perform the task well (eg, frontline nurses with many other responsibilities) may be counterproductive compared with training a few personnel with time dedicated to this task; and (3) training existing personnel in history-taking may have been used to delay the necessary hiring of more staff to take BPMHs. Future studies could address several of these shortcomings in both the design and implementation of medication history-training intervention components.

Several reasons may explain the association we found between implementing a new EHR and increased rates of discrepancies. Based on mentors' experiences, we suspect it is because sitewide EHR implementation requires significant resources, time, and effort. Therefore, sitewide EHR implementation pulls attention away from a focus on medication safety. Most large vendor EHRs have design flaws in their medication reconciliation modules, with the overarching problem being that their systems are not designed for an interdisciplinary team approach to medication reconciliation (unpublished material). In addition, problems may also exist with the local implementation of these modules and the way they are used by clinicians (eg, bypassing critical steps in the medication reconciliation process that lead to new medication errors). We have updated the MARQUIS toolkit to include pros and cons of EHR software and ideal features and functions of medication reconciliation information technology. We should note that this finding contrasts with previous studies that showed beneficial effects of dedicated medication reconciliation applications, which used proprietary technology, often combined with process redesign, in a focused $\mathrm{Q}$ l effort. ${ }^{10-13}$ These findings suggest the need for improvements in the design, local customization, and use of medication reconciliation modules in vendor EHRs.
Our study has several limitations. We conducted an on-treatment analysis, which may be confounded by characteristics of sites that chose to implement different intervention components; however, we adjusted for sites in the analysis. Some results are based on a limited number of sites implementing an intervention component (eg, defining roles and responsibilities). Although this was a longitudinal study, and we adjusted for seasonal effects, it is possible that temporal trends and cointerventions confounded our results. The adjudication of discrepancies for the potential for harm was somewhat subjective, although we used a rigorous process to ensure the reliability of adjudication, as in prior studies.,14 As in the main analysis of the MARQUIS study, this analysis did not measure intervention fidelity.

Based on these analyses and the literature base, we recommend that hospitals focus first on hiring and training dedicated staff (usually pharmacists) to assist with medication reconciliation at discharge. ${ }^{7}$ Hospitals should also be aware of potential increases in medication discrepancies when implementing a large vendor EHR across their institution. Further work is needed on the best ways to mitigate these adverse effects, at both the design and local site levels. Finally, the effect of medication history training on discrepancies warrants further study.

Disclosures: SK has served as a consultant to Verustat, a remote health monitoring company. All other authors have no disclosures or conflicts of interests.

Funding: This study was supported by the Agency for Healthcare Research and Quality (grant number: R18 HS019598). JLS has received funding from (1) Mallinckrodt Pharmaceuticals for an investigator-initiated study of opioid-related adverse drug events in postsurgical patients; (2) Horizon Blue Cross Blue Shield for an honorarium and travel expenses for workshop on medication reconciliation; (3) Island Peer Review Organization for honorarium and travel expenses for workshop on medication reconciliation; and, (4) Portola Pharma- 
ceuticals for investigator-initiated study of inpatients who decline subcutaneous medications for venous thromboembolism prophylaxis. ASM was funded by a VA HSR\&D Career Development Award (12-168)

Trial registration: ClinicalTrials.gov NCT01337063

\section{References}

1. Cornish PL, Knowles SR, Marchesano R, et al. Unintended medication discrepancies at the time of hospital admission. Arch Intern Med. 2005;165(4):424 429. https://doi.org/10.1001/archinte.165.4.424.

2. Kaboli PJ, Fernandes $O$. Medication reconciliation: moving forward. Arch Intern Med. 2012;172(14):1069-1070. https://doi.org/10.1001/archinternmed.2012.2667.

3. Schnipper JL, Mixon A, Stein J, et al. Effects of a multifaceted medication reconciliation quality improvement intervention on patient safety: final results of the MARQUIS study. BMJ Qual Saf. 2018;27(12):954-964. https://doi. org/10.1136/bmjqs-2018-008233.

4. Salanitro AH, Kripalani S, Resnic J, et al. Rational and design of the Multicenter Medication Reconciliation Quality Improvement Study (MARQUIS). BMC Health Serv Res. 2013;13:230. https://doi.org/10.1186/1472-6963-13230.

5. Mueller SK, Kripalani S, Stein J, et al. Development of a toolkit to disseminate best practices in inpatient medication reconciliation. Jt Comm J Qual Patient Saf. 2013;39(8):371-382. https://doi.org/ 10.1016/S1553-7250(13)39051-5.

6. Maynard GA, Budnitz TL, Nickel WK, et al. 2011 John M. Eisenberg patient safety and quality awards. Mentored implementation: building leaders and achieving results through a collaborative improvement model. Innovation in patient safety and quality at the national level. Jt Comm J Qual Patient Saf. 2012;38(7):301-310. https://doi.org/10.1016/S1553-7250(12)38040-9.

7. Mueller SK, Sponsler KC, Kripalani S, Schnipper JL. Hospital-based medication reconciliation practices: a systematic review. Arch Intern Med. 2012;172(14):1057-1069. https://doi.org/10.1001/archinternmed.2012.2246.

8. Vogelsmeier A, Pepper GA, Oderda L, Weir C. Medication reconciliation: a qualitative analysis of clinicians' perceptions. Res Social Adm Pharm. 2013;9(4):419-430. https://doi.org/10.1016/j.sapharm.2012.08.002.

9. Kaboli PJ, Hoth AB, McClimon BJ, Schnipper JL. Clinical pharmacists and inpatient medical care: a systematic review. Arch Intern Med. 2006;166(9):955964. https://doi.org/10.1001/archinte.166.9.955.

10. Plaisant C, Wu J, Hettinger AZ, Powsner S, Shneiderman B. Novel user interface design for medication reconciliation: an evaluation of Twinlist. J Am Med Inform Assoc. 2015;22(2):340-349. https://doi.org/10.1093/jamia/ ocu021.

11. Bassi J, Lau F, Bardal S. Use of information technology in medication reconciliation: a scoping review. Ann Pharmacother. 2010;44(5):885-897. https:// doi.org/10.1345/aph.1M699.

12. Marien S, Krug B, Spinewine A. Electronic tools to support medication reconciliation: a systematic review. J Am Med Inform Assoc. 2017;24(1):227-240. https://doi.org/10.1093/jamia/ocw068.

13. Agrawal A. Medication errors: prevention using information technology systems. Br J Clin Pharmacol. 2009;67(6):681-686. https://doi.org/10.1111/ j.1365-2125.2009.03427.x.

14. Pippins JR, Gandhi TK, Hamann C, et al. Classifying and predicting errors of inpatient medication reconciliation. J Gen Intern Med. 2008;23(9):1414-1422. https://doi.org/10.1007/s11606-008-0687-9. 